\title{
Neues vom Übermenschen
}

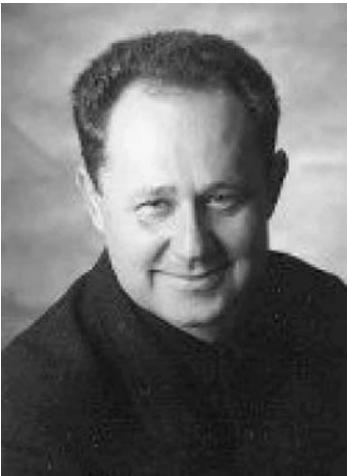

Prof. Dr. med. Manfred Wildner
Bibliografie

DOI http://dx.doi.org/

10.1055/s-0032-1331750

Gesundheitswesen 2013;

75: 769-770

(c) Georg Thieme Verlag KG

Stuttgart · New York

ISSN 0941-3790

Korrespondenzadresse

Prof. Dr. med. Manfred Wildner

Bayerisches Landesamt für Gesundheit und Lebensmittelsicherheit

Veterinärstraße 2

85762 Oberschleißheim

manfred.wildner@|gl.bayern.de
Der schöne Frauenname Olympia ist griechischen Ursprungs. Er bedeutet „Vom Olymp“, was sich in freier Wiedergabe auch als „die Himmlische“ übersetzen ließe. Bekannt wurde Olympia durch das so benannte Zeusheiligtum im Nordwesten der Halbinsel Peloponnes: für über 1000 Jahre Austragungsort der Olympischen Spiele der Antike. Die Sieger - ihre Namen sind seit 776 v. Chr. dokumentiert - wurden mit einem Kranz aus Olivenzweigen geehrt, als von den Göttern begünstigte Menschen. Dieses himmelwärts gerichtete, sich selbst übersteigende menschliche Streben findet sich auch bei den Olympischen Spiele der Neuzeit, welche erstmals 1896 in Athen abgehalten wurden: Citius, altius, fortius (lateinisch: Schneller, höher, stärker) ist das offizielle Motto der olympischen Bewegung.

Diesem Motto macht die sportliche Entwicklung seither alle Ehre: die körperliche Selbst-Transzendenz erreicht fast 9 Meter im Weitsprung, 100 Meter-Sprintzeiten unter $10 \mathrm{~s}$, über $200 \mathrm{Kg}$ im Reißen und Stoßen bei den himmelsstürmenden Männern. Und bei den Frauen? Hier imponieren ganz besonders die himmlischen Fähigkeiten im Wasser: 15- und 16-jährige Mädchen, welche sich an die Weltspitze schwimmen und im direkten Vergleich teilweise sogar die männlichen Olympioniken hinter sich gelassen hätten. Olympia ist wahrhaftig weiblich. Dabei versprechen die Olympioniken seit Sydney 2000 im olympischen Eid, sich „einem Sport ohne Doping und Drogen (zu) verpflichten“. Sehen wir hier MenschlichÜbermenschliches oder Menschlich-Allzumenschliches? Gar eine Spielart „Olympischer Medizin“ [1]? Oftmals ist ja das Dementi der erste Schritt der Wahrheitsoffenbarung, dem Himmel sei's geklagt.

Dabei ist das Streben nach Besserung, nach Vervollkommnung eine uralte Triebfeder einzelner Menschen und menschlicher Gemeinschaft. Sind es nicht gerade die Höchstleistungen, die uns in den verschiedensten Lebensbereichen, in Handwerk und Kunst, in Wissenschaft und Technik so manches Hilfreiche, Angenehme und Heilsame beschert haben? Bach und Beethoven, Dürer und Da Vinci, Galileo und Gauss, Kolumbus und Cook, Teilhard und Tutu, Volterra und Watt: Leben wir nicht in erheblichem Ausmaß von solchen einsamen Spitzenleistungen, durch die Einzelne oftmals unter erheblichen persönlichen Opfern Großes geschaffen und der Menschheit Großes hinterlassen haben? Gilt dies nicht auch für die Heilkunde mit ihren großen Namen: Hippokrates und Avicenna, Paracelsus und De Quesnay, Ignaz Semmelweiss und Joseph Lister, Robert Koch und Max von Pettenkofer, Siegmund Freud und Emil
Kraepelin, William T. G. Morton und William S. Halsted, Ferdinand Sauerbruch und Christian Barnard, Albert Schweitzer und Medecins sans frontiéres als kleine, unvollständige Auswahl. Nicht zu vergessen die lange Liste der Nobelpreisträger und auch der herausragenden Forscher und Persönlichkeiten, die wie Wilhelm Röntgen oder Florence Nightingale als Nicht-Ärzte die Medizin richtungweisend beeinflusst haben. Sicher war bei diesen Höchstleistungen auch selbstvergessene Freude am schöpferischen bzw. hingebungsvollen Tun beteiligt, im Sinne der „Flow“-Erfahrung Csikszentmihalyis [2]. „Göttlich ist es, den Schmerz zu lindern“ - dieser dem griechischen Arzt Galen zugeordnete Ausspruch ist bereits ein Schritt hin auf die „Halbgötter in weiß“, sichtlich eine Form des Übermenschen. Bei genauerem Hinsehen verortet dann auch die altgriechische Mythologie Asklepios, den Gott der Heilkunst, als Halbgott: geboren als Sohn des göttlichen Apollon und seiner menschlichen Geliebten Koronis. Wie die Geschichte genauer geht? Koronis wurde noch vor der Geburt ihres (halb)göttlichen Kindes von der Göttin Artemis getötet, der Schwester des Apollon und Hüterin der Familie und Kinder, weil Koronis sich noch während der Schwangerschaft mit einem Sterblichen eingelassen hatte. Der Götterbote Hermes, quasi der Pressesprecher des Olymp, verhinderte den schlimmsten Skandal und rettete den noch ungeborenen Asklepios per Kaiserschnitt aus dem Mutterschoß. Aufgezogen wurde Asklepios vom Heilkundigen Kentauren Cheiron: einem Mischwesen aus Pferd und Mensch, in seiner Weisheit und Gerechtigkeit weit über allen Lebewesen stehend. Dieser, der also sowohl um die biologischtierische Seite der Existenz weiß, als auch um die geistige Seite, leistete die Aus-, Weiter- und Fortbildung des Asklepios zum Arzt. Cheiron ist seit seinem Tod als Sternbild des Zentauren im südlichen Sternenhimmel zu bewundern.

Um den Bezug zum heutigen Gesundheitswesen in allgemeiner Form deutlich zu machen: Nehmen nicht grundsätzlich Ärztinnen und Ärzte allein durch ihre beruflichen Rollen eine hervorgehobene Stellung ein? Als Helfer und Beistand in kritischen Lebensphasen, als Kompetenzträger in körperlichen, seelischen und sozialen Nöten, als (bisweilen letzte) Hoffnungsträger? Der Arzt als Übermensch - eine uralte Sehnsucht, vielleicht mehr bei den Leidenden als beim Arzt selbst. Vielleicht auch eine beidseitige Allianz: im Heilen und Lindern, im Begleiten hin zum Tod. Womöglich in Übersteigerung der tradierten ärztlichen Rolle auch im gemeinsamen Streben nach der menschlichen (Selbst-)Vervollkommnung: Ästhetisch-chirurgisch und medikamentös das 
Leistungsvermögen von Körper und Geist steigernd für die Olympiaden des Alltags.

Finden sich nicht auch vielerlei subtile Formen dieses (Selbst-) Anspruchs in der ärztlichen Profession? Eminenz statt Evidenz: Das im deutschsprachigen Kulturraum vergleichsweise ausgeprägte Chefarztsystem überrascht besonders die Kollegen, welche hierarchiefreiere Kollegialsysteme kennen und schätzen gelernt haben, beispielsweise im englischen Sprachraum. Und mancher Widerstand gegen eine konsequente evidenzbasierte Medizin mag seinen Ursprung in nostalgischen Arztbildern haben, welche dem wohlwollenden Paternalismus und einer unkritisch überschätzten Therapiefreiheit huldigen. Zumindest beschleicht einen dieser Verdacht bei so mancher Kritik-immuner Argumentation für eine individualisierte, auf den persönlichen Einzelfall unter Vernachlässigung empirischer Evidenz konzentrierte Ausübung der Heilkunde, nicht nur bei Ärztinnen und Ärzten.

Dass der Arztberuf mit besonderen gesellschaftlichen Erwartungen versehen ist, zieht sich durch die Geschichte und ist auch über kulturelle Grenzen hinweg anzutreffen. Die Kehrseite dieser hervorgehobenen sozialen Stellung ist der hohe Selbst- und Fremdanspruch an die Träger dieser beruflichen Rollen: eine Ansprechbarkeit des Tags wie in der Nacht wird eingefordert, welche sich nicht an den Biorhythmen der Ärztin oder des Arztes orientiert, sondern am Auftreten von Krankheit, Schmerz und Leid. Die besonderen ethischen Verpflichtungen finden sich auch in der wohl ältesten Berufsethik, nämlich die des ärztlichen Berufs, wieder und haben ihre Ausdrucksformen durch die Zeiten, vom hippokratischen Eid bis zur ärztlichen Berufsordnung und zum ärztlichen Berufsrecht gefunden.

Gleichzeitig ist seit der Mitte des 20. Jahrhunderts eine mehr oder weniger stille Revolution zu beobachten, welche durch die Bildungsoffensive und durch die breite Verfügbarkeit von Information, nicht zuletzt über das World Wide Web, ausgelöst wurde. Die klassische Informations-Asymmetrie zulasten der Patienten ist deutlich verringert, das Selbstverständnis der Patientinnen und Patienten im Rahmen eines „Empowerments“ verändert mit einem gewachsenen, „gesunden“ Selbstverständnis, während gleichzeitig im Rahmen eines gesamtgesellschaftlichen und weltweiten Demokratisierungsprozesses Hierarchien zunehmend abflachen. Dies hat Folgen sowohl für das individuelle Verhältnis zwischen Ärzten und Ärztinnen mit ihren Patienten und Patientinnen als auch für das Gesundheitswesen als System mit seinen vielfältigen Interaktionen.

So werden vermehrt auch diagnostische und therapeutische Fehlschläge breiter diskutiert: nicht nur bei Medikamenten wie Sulfanilamid, Thalidomid, Rosiglitazon oder Rofecoxib, sondern auch bei bestimmten Praktiken von Psychoanalyse und Chirurgie, bei individuellen Behandlungsfehlern und individuellem ärztlichem Fehlverhalten wie Bestechlichkeit, Betrug und sexuellem Fehlverhalten [3,4]. Ärztliche Fehler bzw. Qualitätsproblematiken sind inzwischen auch Gegenstand der Forschung. Exemplarisch genannt seien die grundlegenden Studien zu Praxisvariationen und zur systematischen Erfassung unerwünschter Ereignisse [5-7]. Im Bereich der Arzneimittel umfasst dies auch die Thematiken von Antibiotika-Fehlverordnungen und iatrogen bedingten Resistenzentwicklungen und von systematischen Indikations- und Dosisfehlern z.B. in der Benzodiazepinverordnung, bei Multimorbidität und dadurch bedingten Arzneimittelwechselwirkungen und im besonderen bei renalen Funktionsstörungen.
Wissenschaftlichen Erkenntnissen zur medizinischen Versorgung gehen auch die vielfältigen Beiträge dieser Ausgabe nach: zu „Gesundheit 2020“, dem neuen Rahmenkonzept der WHO, zur psychologischen Rehabilitation in der Orthopädie und Kardiologie, zu Nutzen und Kosten des Telemonitorings, zu Berufszielen von Studierenden im praktischen Jahr, zur DekubitusProblematik im stationären Sektor, zu Pflegeberatung, Lebensqualität in der Langzeitpflege und Fixierungsmaßnahmen, zum Dolmetscheranspruch bei Gehörlosigkeit, zu Interdisziplinarität bei seltenen Erkrankungen, zu Pflanzenschutzmitteln und Biozidprodukten in Kleinwasseranlagen, zum Datenschutz bei der Verknüpfung von Primär- und Sekundärdaten und zur Finanzierungsproblematik von Kontrazeptiva bei Frauen in Armut.

Der Begriff des „Übermenschen“ findet sich übrigens nicht erst seit modernen olympischen Superstars und auch nicht erst seit Nietzsche und auf ihn Bezug nehmenden ideologischen Überhöhungen [8]. Der Begriff des „Hyperanthropos“ als Idealmensch wurde bereits im 1. Jahrhundert v. Chr. geprägt (Dionysios von Halikarnassos, *54 v. Chr.). Im Umfeld olympischer Superstars gibt es auch nicht nur die Sieger, sondern auch die zweiten, dritten und weiteren Nachplatzierten: Nach alten Überlieferungen reisten in der Antike schon die Zweitplazierten der Olympischen Spielen nur auf Schleichwegen wieder zurück in ihre Heimat. Vermeintliches Übermenschentum generiert immer auch vermeintliches Untermenschentum. Beides ist zu vermeiden. Vielleicht dem Himmel sei gedankt - liegt ein verborgener Sinn für manchen Skandal in der Medizin darin: Dass nämlich Ärzte und Ärztinnen als kostbare Ressource, als hochqualifizierte Expertiseträger und sozial legitimierte Träger weitreichender Entscheidungsbefugnisse sich vor solchen Überhöhungen hüten. Und in diesem Mut zu Demut und gelebter Mitmenschlichkeit dann tatsächliche menschliche Größe und Lebenserfüllung finden - mit oder ohne literarische Ratgeber und nicht nur zur Weihnachtszeit [9-11].

\section{Literatur}

1 Jones DS. Olympic Medicine. N Engl J Med 2012; 367: 289-292

2 Csikszentmihalyi M. Das Flow-Erlebnis. Jenseits von Angst und Langeweile im Tun aufgehen. 8., unv. Aufl. (Übers., Beyond Boredom and Anxiety - The Experience of Play in Work and Games, 1975) Klett Verlag; Stuttgart: 2000

3 Avorn J. Two centuries of assessing drug risks. N Engl J Med 2012; 367: 193-197

4 Annas GJ. Doctors, patients, and lawyers - two centuries of health law. N Engl J Med 2012; 367: 445-449

5 Wennberg J, Gittelsohn A. Small area variations in health care delivery. Science 1973; 182: 1102-1108

6 Brennan TA, Leape LL, Laird NM et al. Incidence of adverse events and negligence in hospitalized patients: Results from the Harvard Medical Practice Study I. New Engl J Med 1991; 324: 370-376

7 Leape LL, Brennan TA, Laird NM et al. The nature of adverse events in hospitalized patients: Results from the Harvard Medical Practice Study II. New Engl J Med 1991; 324: 377-384

8 Nietzsche F. Also sprach Zarathustra. Ein Buch für Alle und Keinen (Privatdruck, 1883-1885). URL: http://www.zeno.org/Philosophie/M/ Nietzsche, + Friedrich/Also + sprach +Zarathustra download 24.08.2012

9 Rohr R, Ebert A. Das Enneagramm: Die 9 Gesichter der Seele (46. Auflage). Claudius Verlag; München: 2008

10 Rosenberg MB. Gewaltfreie Kommunikation (9. überarb. und erw. Neu Auflage). Junfermann. Paderborn 2010

11 Beck D, Cowan C. Spiral Dynamics: Mastering Values, Leadership, and Change. Blackwell Business; Cambridge, Mass., USA: 1996 (dt.: Spiral Dynamics - Leadership, Werte und Wandel. Bielefeld, Kamphausen, 2007 